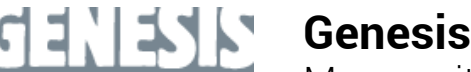

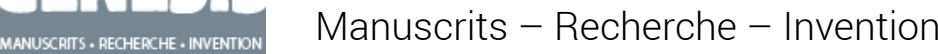

36 | 2013

Proust, 1913

\section{Bidou, Bergotte, la Berma et les Ballets russes}

Une enquête génétique

Nathalie Mauriac Dyer

\section{CpenEdition}

Journals

Édition électronique

URL : https://journals.openedition.org/genesis/1144

DOI : $10.4000 /$ genesis. 1144

ISSN : 2268-1590

Éditeur :

Presses universitaires de Paris Sorbonne (PUPS), Société internationale de génétique artistique littéraire et scientifique (SIGALES)

\section{Édition imprimée}

Date de publication : 15 juin 2013

Pagination : 51-62

ISBN : 978-2-84050-893-9

ISSN : $1167-5101$

\section{Référence électronique}

Nathalie Mauriac Dyer, «Bidou, Bergotte, la Berma et les Ballets russes », Genesis [En ligne], 36 | 2013, mis en ligne le 15 juin 2015, consulté le 11 janvier 2023. URL : http://journals.openedition.org/genesis/ 1144 ; DOl : https://doi.org/10.4000/genesis.1144 


\section{Bidou, Bergotte, la Berma et les Ballets russes Une enquête génétique}

Nathalie Mauriac Dyer

L 'écriture de Proust est, profondément, oblique et allusive. Non qu'elle recherche forcément la connivence avec certain lecteur complice - le terme d'allusion n'est donc qu'à demi satisfaisant -, mais parce qu'un des modes de son élaboration est la métabolisation des références culturelles : défaites et métamorphosées, elles sont restituées sous une forme parfois méconnaissable, moins cryptées (et donc qu'il suffirait de décoder) que cryptiques, c'est-àdire opacifiées. Pour le lecteur, c'est parfois un léger trouble, une étrangeté lexicale ou sémantique locale une « agrammaticalité », dirait Michael Riffaterre - qui permet de soupçonner l'absorption d'un élément étranger par le texte. Le processus est d'autant plus déroutant peut-être qu'il n'exclut pas, simultanément, la tenue à partir des mêmes références d'un discours romanesque explicite. Le fait vaudrait pour toute manifestation artistique ou culturelle dont Proust fut le témoin, mais qui s'intéresse, par exemple, à l'empreinte laissée par les Ballets russes dans le texte de la Recherche ne saurait se limiter à en dresser l'inventaire à partir des seuls index des volumes publiés, ou même de celui des cahiers de brouillon 1 .

Comment et où, dans ces conditions déroutantes, conduire l'enquête génétique ? En effet plus le texte qu'on peut dire « source » (texte-prétexte ou texte-tremplin) est peu diffusé et donc peu accessible, autrement dit : plus il s'éloigne du corpus des grandes œuvres canoniques que Proust a amplement incorporées, récrites et transmuées, mais qui nous sont encore familières (comme celles de Balzac, Flaubert ou Baudelaire) -, moindres sont nos chances de le découvrir. Ainsi, ce n'est que relativement récemment qu'a été mise à jour l'importance génétique d'ouvrages de critique d'art appartenant à des collections de vulgarisation (publiées au début du siècle et jamais rééditées), ouvrages que Proust consommait de manière systématique et où il a largement pioché2. Parmi les publications moins diffusées ou littéralement éphémères, le corpus des revues (politiques, historiques, littéraires et artistiques) et surtout celui (plus massif encore) de la presse quotidienne restent de véritables défis, en même temps que des mines ${ }^{3}$, pour la recherche génétique. Dans le cas des Ballets russes, c'est évidemment ce dossier qu'il faudrait dépouiller en priorité, et systématiquement, pour les années 1909-1914. Or le chercheur qui se livre à ce travail ne doit pas privilégier les passages explicitement dévolus au commentaire des « Russes » (puisque, je l'ai dit, les références se dispersent tout en s'opacifiant), et, tout en gardant en mémoire vive l'ensemble du texte romanesque, se rendre attentif à des phénomènes de surimpressions partielles, troubles en quelque sorte. Pour créer les conditions d'une sérendipité maximale, la mémoire du texte devrait, idéalement, se doubler de celle des avanttextes. Fort heureusement, leur connaissance de plus en plus intégrale et systématique - telle qu'elle se développe actuellement grâce à l'édition des cahiers de brouillon nous épargne à elle seule, parfois, quelques détours.

Mais il ne suffit pas, bien entendu, de lire les cahiers, car il serait illusoire de penser que le processus de métabolisation s'y donne à voir dans quelque transparence native (l'allusion, l'image cryptique se manifestent d'emblée telles quelles, ne faisant ensuite que se préciser, se renforcer ou s'atténuer, secréter un réseau, parfois

1. Voir Akio Wada, Index général des cahiers de brouillon de Marcel Proust, Osaka, Graduate School of Letters, 2009.

2. Voir Kazuyoshi Yoshikawa, Proust et l'art pictural, Paris, Champion, 2010.

3. Voir Hiroya Sakamoto, «Quelques allusions à la presse dans les cahiers de la Guerre », BIP, no 42, 2012. 
se recontextualiser). Pourtant il peut arriver que Proust mentionne l'ouvrage, la revue, l'article ou, dans le cas qui nous occupe, l'auteur et le sujet, qui a été (ou sera) associé par divers phénomènes d'hybridation textuelle partielle au processus rédactionnel. L'occurrence que je prendrai ici sera donc le point de départ d'un trajet dans les brouillons. Trajet au cours duquel on s'efforcera de repérer et mesurer dans toute leur épaisseur temporelle les phénomènes de métamorphose et d'appropriation d'un texte « étranger». On verra d'ailleurs que la temporalité de l'indice ne coöncide pas forcément avec celle du processus (mais la présentation suivra ici l'ordre de la recherche, et non celui de la genèse). On constatera qu'au cours du travail rédactionnel Proust fait subir au texte étranger ou externe le sort que subissent souvent ses propres fragments ou unités rédactionnelles, à savoir l'éclatement et la dissémination. La diversité des contextes d'inscription de la référence ainsi reconfigurée conduira à s'interroger in fine sur la ou les intentionnalités qui pourraient la sous-tendre : de quels discours obliques pourraient relever, dans notre exemple, le travestissement et la transposition des références?

Je partirai d'un extrait de La Prisonnière ${ }^{4}$, tome publié à titre posthume (1923) et qui n'a été qu'irrégulièrement revu par Proust : la rédaction du passage retenu remonte à la fin de 1915 ou au début de 1916, dans le Cahier dit de «mise au net » XI. Le contexte est celui de la soirée Verdurin, dominée par deux « exécutions », celle, musicale, du septuor posthume de Vinteuil, décisive dans l'initiation esthétique du protagoniste, et celle, mondaine, de M. de Charlus, brutalement chassé du salon ${ }^{5}$. Le baron ayant en effet imprudemment humilié les ambitions aristocratiques de la Patronne, elle se venge en montant contre lui son amant et protégé, le violoniste Morel :

«Laissez-moi je vous défends de m'approcher, cria Morel au baron. Vous ne devez pas être à votre coup d'essai, je ne suis pas le $1^{\text {er }}$ que vous essayez de pervertir ! » Ma seule consolation du était que j'allais voir Morel et les Verdurin pulvérisés par M. de Charlus. [...] Or il se produisit cette chose extraordinaire [...] dans ce salon qu'il dédaignait, ce grand seigneur (à qui n'était pas plus inhérente essentiellement inhérente la supériorité sur les roturiers qu'elle ne le fut à tel de ses ancêtres angoissés devant le tribunal révolutionnaire,) ne sut $[\ldots]$ que balbutier : «Qu'est-ce que cela veut dire, qu'est-ce qu'il y a. » On ne l'entendait même pas. <Et la pantomime éternelle de la terreur panique a si peu changé qu'aujourd'huieneore [que] ce vieux Monsieur à qui il arrivait dans un une aventure désagréable dans un salon parisien répétait à son insu les quelques attitudes schématiques où la plus arehä̈que seulpture greeque dans lesquelles la sculpture grecque des premiers âges stylisait une fuite de l'épouvante des nymphes poursuivies par le Dieu Pan.> (Cahier XI, fo 30 et papiers collés, fo 31 et marge ; voir $P$, III, p. 820-821).

La chute, en fin de paragraphe, est suivie d'une longue digression anticipatrice rédigée sur une paperole, à la fin de laquelle Proust reprend l'image frappante : «Tandis que M. de Charlus, assommé <sur le coup $>$ par les paroles de <que venait de prononcer $>$ Santois [Morel] et l'attitude de la Patronne faisait les prenait la pose épouvantée de la nymphe en proie à la terreur pani[que] [...] » (Cahier XI, paperole au folio 31 ; voir $P$, III, p. 823). Frappé par le burlesque de la métamorphose en nymphe du vieil inverti6, en un de ces retournements carnavalesques qu'aime ménager l'écriture proustienne, le lecteur ne s'arrête pas aux « quelques attitudes schématiques dans lesquelles la sculpture grecque des premiers âges stylisait l'épouvante des nymphes poursuivies par le Dieu Pan ». C'est pourtant une pseudoréférence ${ }^{7}$ puisque, contrairement à ce que prétend le narrateur, Pan pourchasseur de nymphes n'est pas un thème de la sculpture grecque archaïque, mais un motif littéraire tardif, popularisé à Rome par les Métamorphoses d'Ovide8. Sauf à buter sur la légère "agrammaticalité », le lecteur passe sans s'attarder ; la poursuite de la nymphe Syrinx par Pan étant au moins un topos de la peinture occidentale, la référence semble plausible.

4. Une première version de cette enquête génétique a été présentée le 9 avril 2010, lors d'une journée d'étude organisée par Pierre-Edmond Robert au Centre de recherches proustiennes de l'Université Paris III-Sorbonne nouvelle.

5. Le texte joue bien sur le double sens : «Il vaudrait mieux retarder cette exécution [celle de Charlus] jusqu'après celle des morceaux », estime Mme Verdurin, soucieuse de ne pas troubler Morel ( $P$, III, p. 749).

6. Voir Martine Reid, « Exécution (Proust)», dans Terreur et représentation, dir. Pierre Glaudes, Grenoble, ELLUG, 1996, p. 236.

7. Pierre-Edmond Robert, seul éditeur de La Prisonnière qui se soit risqué à annoter, ne fournit aucune référence concluante : voir $P$, III, p. 821, n. 1 . 8. I, vers 689-746. 


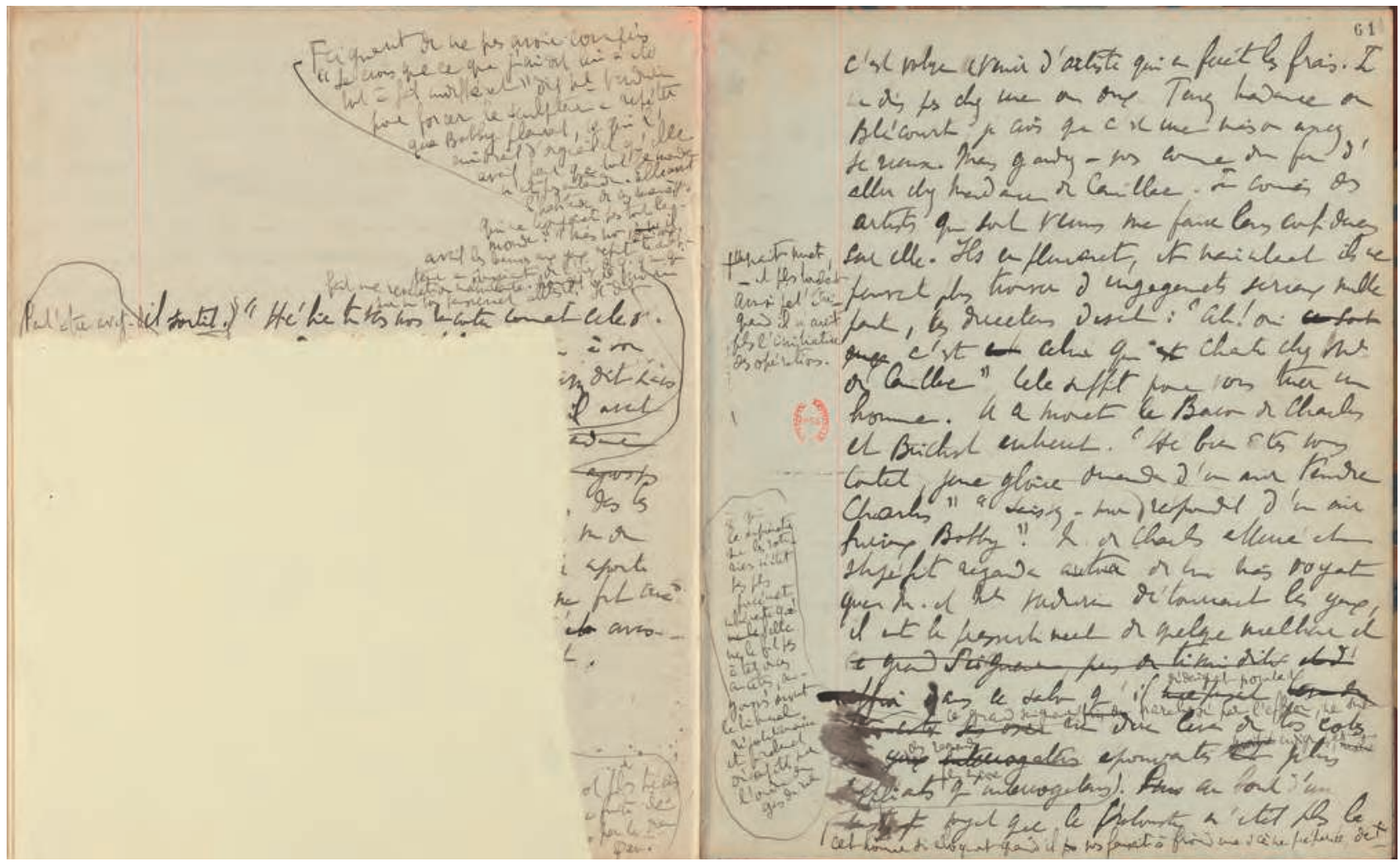

Fig. 1 : Cahier 73, fos 60 vo-61 ro (BnF, NAF 18323)

C'est donc dans les marges d'une autre recherche (en prélude à l'établissement du fac-similé critique du Cahier 73 pour l'édition des Cahiers 1 à 75 de la $B n F$ ) qu'est apparue la note de régie qui fait rebondir la lecture en rouvrant la perspective génétique. Ce cahier fait en effet partie de ceux auxquels manquent un certain nombre de feuillets (ou fragments de feuillets), qu'un Proust de plus en plus pressé a pendant la Guerre, à l'étape de la « mise au net », arrachés, découpés ou déchirés puis collés pour s'épargner quelques recopiages. Afin de fournir au lecteur une version aussi intelligible que possible et de rendre compte du dynamisme de la genèse, l'éditeur doit retrouver ces fragments, les identifier de manière certaine et les restituer à leur place originelle avant de procéder à leur transcription ${ }^{9}$.

Le Cahier 73, ainsi dépecé au profit des Cahiers IX, X et XI, est le seul brouillon connu de la soirée Verdurin. Il prend fin précisément sur l'exclusion de Charlus ; la scène commence à la dernière page recto du cahier (fo 61 ro) et se poursuit sur le verso en vis-à-vis. Or ce folio 60 vo est lacunaire (voir fig. 1). Il n'a pas été difficile de retrouver le fragment manquant dans le Cahier XI, au folio 26 ro, où il correspond à la face envers du fragment collé « en plein » sur la page. Les restaurateurs de la Bibliothèque nationale l'ayant en partie dégagé (fig. 2), il ne restait plus qu'à reconstituer numériquement le feuillet (fig. 3), à le déchiffrer et à le transcrire. On y trouve le premier jet de notre passage :

9. Sur les modalités et les problèmes posés par cette restitution, voir mon article « La reconstitution des cahiers de brouillon du fonds Proust : points de méthode et principes de foliotation complémentaire », BIP, no 38, 2008, p. 99-105. Le Cahier 73 a été transcrit sous le protocole diplomatique de l'édition Brepols par Shuji Kurokawa, et il est en cours de relecture. 


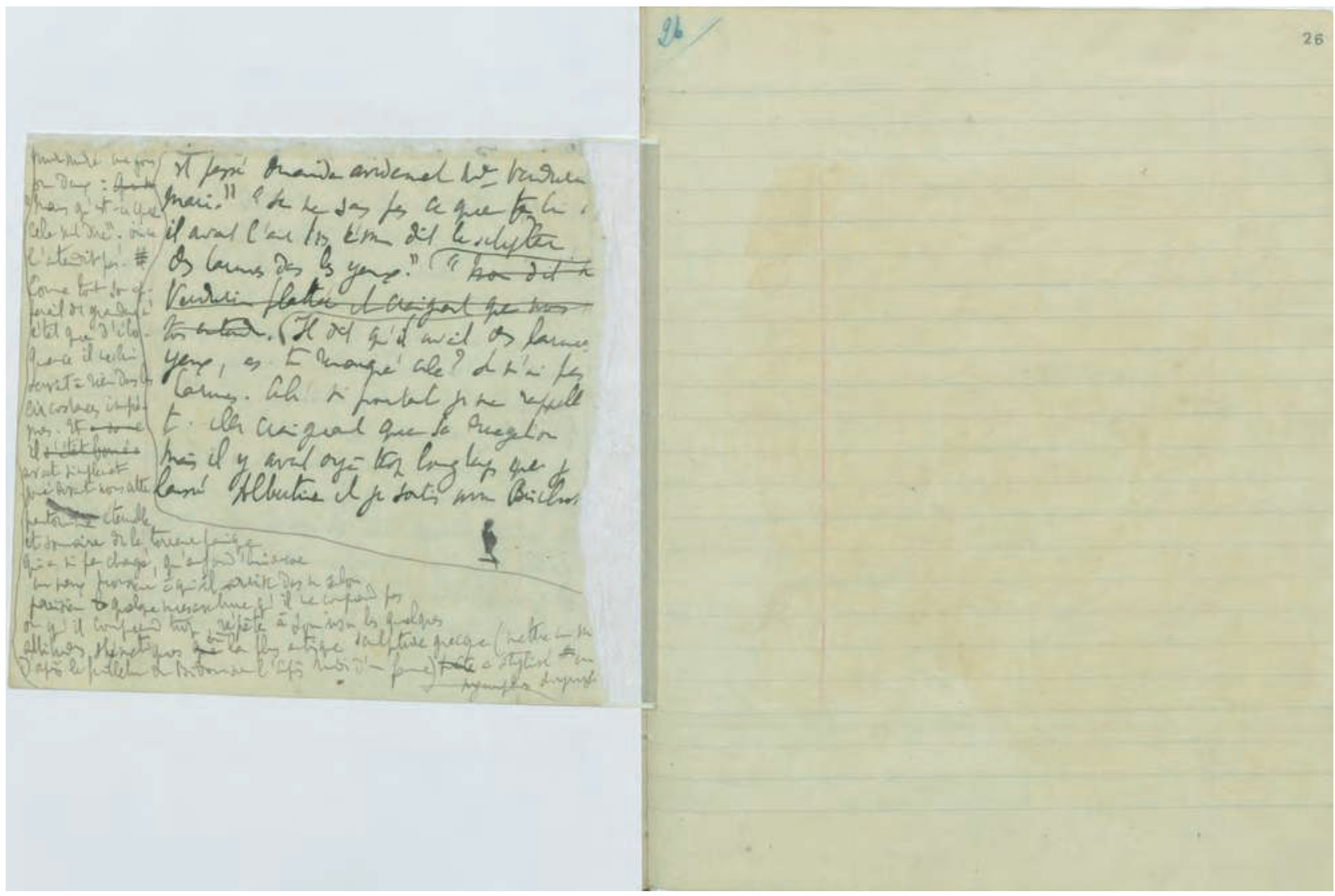

Fig. 2 : Cahier XI, envers du papier collé au folio 26 ro (BnF, NAF 16718)

Et en somme il [M. de Charlus] s'était borné à avait simplement joué devant nous cette pantomime éternelle et sommaire de la terreur panique qui a si peu changé, qu'aujourd'hui encore un vieux monsieur à qui il arrive dans un salon parisien ta quelque mésaventure qu'il ne comprend pas ou qu'il comprend trop, répète à son insu les quelques attitudes schématiques que où la plus antique sculpture grecque (mettre un mot plus précis d'après le feuilleton de Bidou sur l'Après midi d'un faune) prête a stylisé une fuite de nymphes surprises par le Dieu Pan (Cahier 73, fo 60-60b vo).

« (mettre un mot plus précis d'après le feuilleton de Bidou sur l'Après midi d'un faune) »: la parenthèse de régie condense l'information que le lecteur du roman n'est pas censé connaître, à savoir que l'image agrammaticale procède - au moins en partie et selon des modalités qui restent à définir - d'un article du critique Henry Bidou (1873-1943). Il s'agit du feuilleton «L'Après-midi d'un faune et l'esthétique de M. Nijinski10 », consacré au «tableau chorégraphique » créé dans le cadre de la quatrième saison des Ballets russes au théâtre du Châtelet au printemps de 1912, avec la musique composée par Debussy sur le poème de Mallarmé du même nom¹1, dans un décor et des costumes de Léon Bakst.

10. Journal des Débats, « La Semaine dramatique », 10 juin 1912, p. 1-2. 11. Prélude à l'après-midi d'un faune avait été composé par Debussy en 1894 d'après le poème de Mallarmé, L'Après-midi d' un faune, publié en plaquette en 1876 avec des illustrations de Manet. 


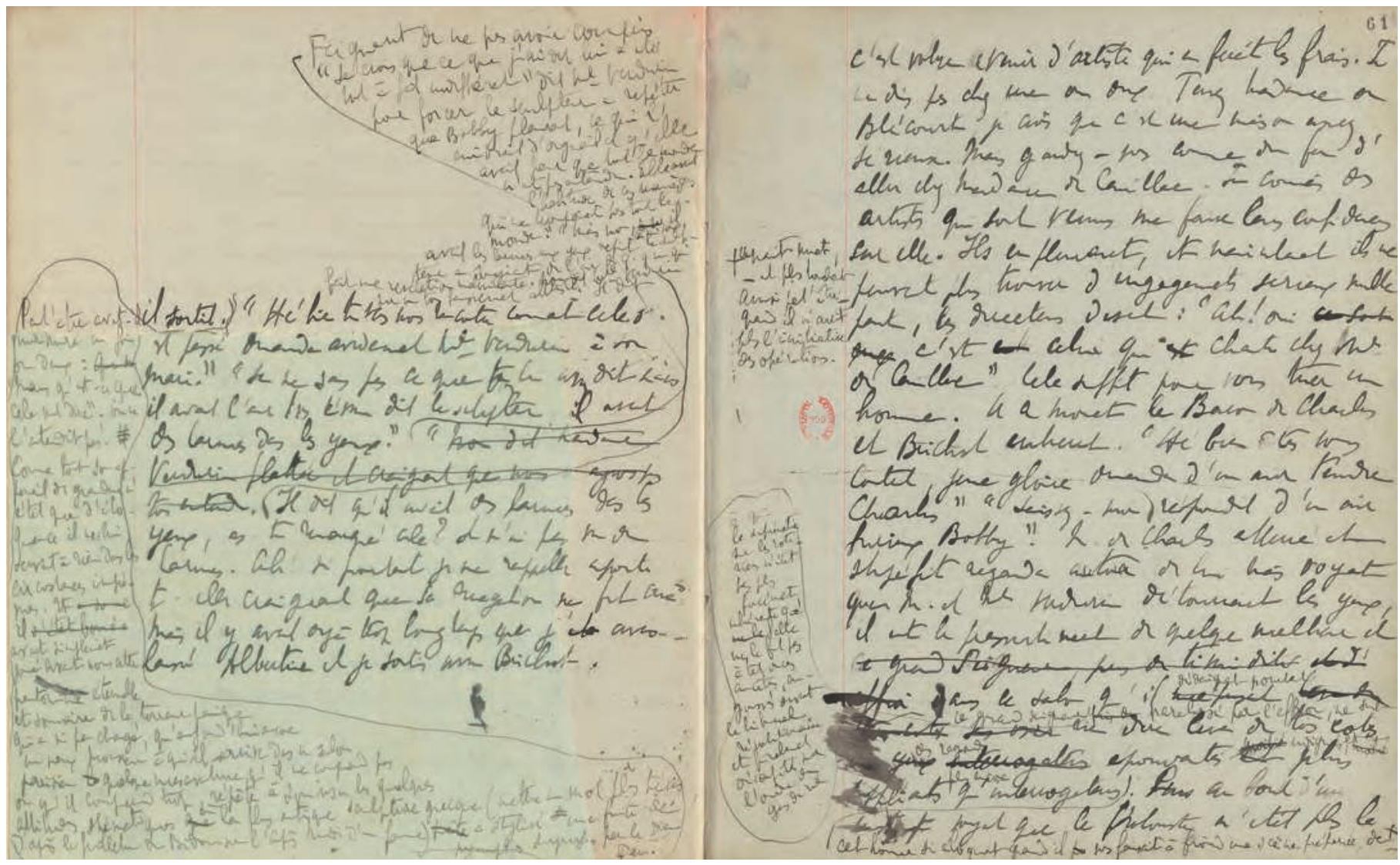

Fig. 3 : Cahier 73, reconstitution du folio $60-60$ b vo et fo 61 ro (BnF, NAF 18323)

La parenthèse de régie du Cahier 73 annonce celle du Cahier 74, relative aux chroniques militaires du même auteur : «(voir les admirables articles d'Henri [sic] Bidou)» (fo 126 vo). Ces articles du Journal des Débats ont en effet nourri en 1917 l'exposé de stratégie de Saint-Loup intégré ensuite au Côté de Guermantes I'2, Proust imputant plus loin au personnage sa propre admiration pour le journaliste13. Cette contribution à la genèse de la Recherche est bien connue, mais on sait moins que, avant la Guerre, de 1911 à 1913, Bidou avait tenu au même Journal des Débats la chronique dramatique hebdomadaire ${ }^{14}$ et surtout que Proust la suivait.

Cet article, extrêmement élogieux, mérite de retenir l'attention (et d'être relu : à ma connaissance du moins, aucune bibliographie de la réception du Faune ne le mentionne). Le «tableau chorégraphique » de Nijinsky avait été donné entre le 29 mai et le 10 juin 191215 : que Bidou ait attendu le matin de la «dernière » pour le publier n'est certainement pas un hasard. Il ne s'agit pas là, semblet-il laisser entendre, de l'article d'un proche, d'un membre

12. Voir $C G I$, II, p. 408, n. 1 ; p. 409, n. 1 ; p. 411 , n. 5 et 6 ; p. 412, n. 2 ; p. 414, n. 1 et 2 .

13. Après la mort de Saint-Loup au front, Gilberte mentionne le nom de Bidou comme celui d' « un critique pour lequel il avait une profonde admiration et qui exerçait visiblement une grande influence sur ses idées militaires » (TR, IV, p. 559).

14. Chroniques réunies sous le titre L'Année dramatique, 1911-1912 et L'Année dramatique, 1912-1913, Paris, Hachette, 1912 et 1913. Voir aussi A. Compagnon, Proust entre deux siècles, Paris, Éditions du Seuil, 1989, p. 87,89 (à propos d'Esther).

15. Répétition générale publique le 28 mai, première le 29 , représentations suivantes les 31 mai, 1er, 3, 5, 7, 8 et 10 juin 1912. 
du «premier cercle », comme ceux de Jean Cocteau et de Jacques-Émile Blanche, respectivement parus la veille et le matin de la « première » 16 ; il ne s'agit pas non plus d'un article hostile, scandalisé et pudibond comme celui publié par Gaston Calmette au lendemain de cette première (30 mai $\left.{ }^{17}\right)$, ni d'une riposte « à chaud » comme celle de Diaghilev ou de Rodin (31 mai 18 ) - d'ailleurs les censeurs l'ont emporté, imposant dès la deuxième représentation une modification du geste final, puisqu'il « convenait de ne pas oublier que "le spectateur français veut être respecté"19 ». Bidou a pris son temps : sa critique sera dépassionnée et « objective », mais aussi ambitieuse, puisqu'il s'agit de réfléchir à l'" esthétique » de Nijinsky.

Dans la première partie, la description précise (et aujourd'hui précieuse) de la chorégraphie - déplacements relatifs des danseurs, rapports, dans les postures, entre frontalité et profils - suggère que le journaliste a suivi attentivement plusieurs représentations :

Le faune [...] était sur son rocher, accroupi de profil, divisant d'une main raide une grappe de raisin, quand les nymphes sont entrées. Il a soudain tourné la tête, observé comme un chat, et d'un pas de velours il est rentré dans la coulisse. Il en sort soudain à l'extrême-gauche, de plein profil, droit, les coudes au corps, les avant-bras un peu levés ; les mains toujours longues, en plan vertical, descendant un peu en oblique. Il se trouve ainsi sur le même rang que les nymphes. Celles-ci l'aperçoivent tout à coup, laissent tomber leur fardeau, et, les genoux pliés, fuient en glissant, comme si elles étaient emportées sur la plaque de verre de la lanterne magique. / Le faune les laisse fuir. Il a aperçu la nymphe du milieu. [...] Sa proie s'échappe et il reste seul, immobile, étonné. Alors, par bandes, pour le railler, les nymphes reviennent et se retirent, toujours dans le même plan ; par dérision, elles agitent en ciseaux leurs bras mécaniques; ou elles fuient dans une posture très singulière, courant si bas qu'elles sont presque agenouillées, le torse de face, une main levée et l'autre sur la hanche. Le faune déçu aperçoit enfin les vêtements laissés à terre. Il les saisit et les emporte agilement sur son rocher. La toile tombe.

Dans la seconde partie qui s'efforce d'élucider quelques « sources antiques » de l'art de Nijinsky, les références suggèrent au moins autant l'érudition du chorégraphe que celle du journaliste :
Parmi les spectateurs, ceux qui ont quelque culture (j'ignore quel est leur nombre) ont d'abord le sentiment qu'ils voient s'animer tout l'art grec archaïque. Il n'est presque pas un mouvement qui n'évoque une œuvre ${ }^{20}$. Cette fuite étonnante de nymphes aux genoux ployés, un bras levé et un poing sur la hanche, c'est une image fameuse ; c'est dans cette pose qu'à la fin du septième siècle, Mikkiadès et Archermos, fils de Mélas, sculptèrent au moment où elle court, une Artémis ailée. Les deux nymphes qui entrent d'abord, la main levée à la hauteur de l'épaule, nous rappellent une Hespéride qui fait le même geste sur une métope d'Olympie. L'aspect des figures est celui des statues de l'Acropole [...]. On a donc très naturellement l'idée que L'Après-midi d' un faune est une curieuse suite d'images du septième siècle et du sixième ${ }^{21}$.

Or la comparaison à l'art grec est déjà un topos de la critique : selon Blanche dans "L'Antiquité en 1912 », le Faune est un «bas-relief archaïque 22 », né d'un « besoin de retour à la tradition classique primitive » après « trois

16. Jean Cocteau, «Une répétition du Prélude à l'Après-midi d' un faune », Comæedia, 28 mai 1912; Jacques-Émile Blanche, «L'Antiquité en 1912 », Le Figaro, 29 mai 1912.

17. Gaston Calmette, «Un faux pas », Le Figaro, 30 mai 1912 : « Nous avons eu un faune inconvenant avec de vils mouvements de bestialité érotique et des gestes de lourde impudeur ».

18. Diaghilev fit publier le 31 mai dans Le Figaro une « Lettre à Serge de Diaghilev » par Odilon Redon, ami de Mallarmé, et une longue citation de l'article dithyrambique publié par Rodin la veille ( La rénovation de la danse », Le Matin, 30 mai 1912).

19. « Fin de l'incident de L'Après-midi d'un faune », Le Gaulois, 1er juin 1912, p. 1. Voir aussi Roland Huesca, Triomphes et scandales. La belle époque des Ballets russes, Paris, Hermann, 2001, p. 141.

20. Bidou avait parlé plus haut des nymphes telle une « frise vivante », ou de leur groupe dessinant un «fronton».

21. Journal des Débats, « La Semaine dramatique », 10 juin 1912, p. 1-2 (p. 1).

22. Le Figaro, art. cité, 29 mai 1912 (chez Cocteau, Comœdia, art. cité, 28 mai 1912, un «bas-relief mobile »; Redon parlait d'une « frise vivante », Le Figaro, 31 mai 1912). Voir aussi Rodin, « La rénovation de la danse », Le Matin, 30 mai 1912 : «Qu'on ne s'étonne pas de voir l'églogue d'un poète contemporain reportée au temps de la Grèce primitive ; cette transposition offrait pour le geste archaïque l'occasion heureuse de se produire au commandement d'une volonté expressive. » On pourra consulter aussi le compte rendu de Hugo von Hofmannsthal sur $L$ 'Aprèsmidi d' un faune, initialement paru dans le Berliner Tageblatt en 1912 et cité dans Nijinsky, "Prélude à L'Après-midi d'un Faune ", éd. J.-M. Nectoux, Paris, Adam Biro, 1989, p. 52-53. 
années de réflexion, de recherches, de conversations et d'étude », car Nijinsky «vit dans les musées et les bibliothèques ». Mais Bidou semble avoir été le premier à chercher les sources précises du chorégraphe23. Sans doute s'efforce-t-il d'autant plus de suggérer le sérieux de son approche qu'il s'aventure (pour la première et d'ailleurs seule fois de ses deux années de feuilletoniste dramatique) sur le terrain chorégraphique. Il rend hommage à Blanche, mais entend aller plus loin :

M. J.-É. Blanche, dans un brillant article, a montré comment ce spectacle nous présentait l'interprétation la plus actuelle de l'Antiquité. Cependant, il y a dans cette étrange pantomime autre chose qu'une restitution ingénieuse. M. Nijinski, quand il s'explique, fait entrevoir les principes d'un art subtil et réfléchi, qui intéresse tous les interprètes tragiques. L'idée essentielle peut s'écrire ainsi : traiter le mouvement comme les poètes traitent la parole [...]. Dans cette élimination toutes les transitions sont supprimées. Ceci est fort intéressant pour les tragédiens. Les nymphes ne voient pas le faune, et tout à coup le voient. Elles sont immobiles et tout à coup elles se déclenchent, et tombent net dans la pose qui représente leur fuite. [...] [Nijinsky] vient à cette conclusion hardie, mais raisonnable et belle, que l'immobilité représente le mouvement mieux que le mouvement même 24 .

La posture des nymphes en fuite, décrites « les genoux pliés » ou «ployés 25 », « courant si bas qu'elles sont presque agenouillées, le torse de face », une main ou un bras « levé et l'autre sur la hanche » correspond approximativement à celle d'une photographie du Faune posée devant 1'objectif du baron Adolf de Meyer, vraisemblablement en $1912^{26}$ (voir fig. 4). Le cliché photographique accentue la ressemblance avec la figure statuaire citée par Bidou, celle de l'Artémis ailée, arborant l'attitude que les Grecs appelaient de la « course agenouillée » (voir fig. 5 et 5 bis). C'est donc dans cette pose caractéristique de la «terreur panique » que le lecteur de la Recherche pourrait, dorénavant, se figurer M. de Charlus.

Proust a effacé le titre de l'œuvre (d'autant moins repérable que le faune est devenu Pan), le nom du chorégraphe et celui du journaliste : M. de Charlus paniqué " répétait à son insu les quelques attitudes schématiques dans lesquelles » Nijinsky, selon Henry Bidou, « stylisait » dans L'Après-midi d'un faune « l'épouvante des nymphes poursuivies par le Dieu Pan »d'après « la sculpture grecque des premiers âges ». Les omissions produisent un court-circuit des rapports : là où chez Bidou un comparé (la posture des nymphes en fuite) appelait des comparants (certaines œuvres grecques), chez Proust le comparé a « glissé » et s'est assimilé aux comparants, autrement dit les nymphes sont « devenues » les œuvres que certains de leurs gestes évoquaient, et Charlus s'est glissé à son tour à la place du comparé laissée vacante.

Pourquoi l'omission systématique de tous les indices? Certes, Proust exploite sans vergogne certains motifs de l'article de Bidou : la formule « une fuite de nymphes » (en tout cas dans la version du Cahier 73), la comparaison avec la statuaire grecque, l'image de la pantomime, plus proche de l'art dramatique que de la danse - mais rien de tout cela n'était bien original. En réalité, les deux scènes ne se superposent en rien. Contrairement au « tableau chorégraphique » de 1912, la scène proustienne (qui couronne dans le Cahier 73 une série d'images de travestissement de Charlus) présente une sorte d'antinymphe (masculine, âgée, peu attrayante) et subvertit la structure du mythe : en effet, sa panique naît précisément de n'être pas, ou plus, désirée par son faune (Morel27) d'être chassée loin de sa vue, et non pas pourchassée par lui. Au renversement des âges et des sexes s'ajoute donc une réécriture parodique. Ce côté «Belle Hélène » de la scène proustienne, mais aussi son profond pathétique,

23. On sait qu'il fréquenta en effet en octobre-novembre 1910 les salles du Louvre, notamment la galerie Campana pour y étudier les peintures sur vases.

24. «L'Après-midi d'un faune et l'esthétique de M. Nijinski », art. cité, 10 juin 1912, p. 2. Voir J. Cocteau, art. cité, 28 mai 1912 : 1'art de Nijinsky nous évoque la nature « en raison inverse du principe niais par quoi certains artistes cherchent le réalisme sans transposition initiale. La peur panique, la terreur panique, [...] la peur panique nous immobilise ».

25. Cocteau avait utilisé le même adjectif : «Elles s'échappent de droite et de gauche, (ce vol de cygnes, non ! de naïades se sauve), les jambes ployées, les mains vers leur fuite, à deux dimensions, courtes, allongées [...] » (art. cité, 28 mai 1912).

26. Adolph de Meyer, Prélude à l'Après-midi d'un faune, Trente phototypies, Paris, Paul Iribe, 1914. Sur cet album, voir L'Après-midi d'un Faune. Mallarmé, Debussy, Nijinsky, catalogue d'exposition, éd. J.-M. Nectoux, Musée d'Orsay, RMN, 1989, p. 31-34.

27. Sur la « faunesse cachée » en l'inverti, et notamment en Félix, un archétype de Morel, voir Cahier 54, fo 5 ro. Le passage peut être daté de la fin de 1913. 


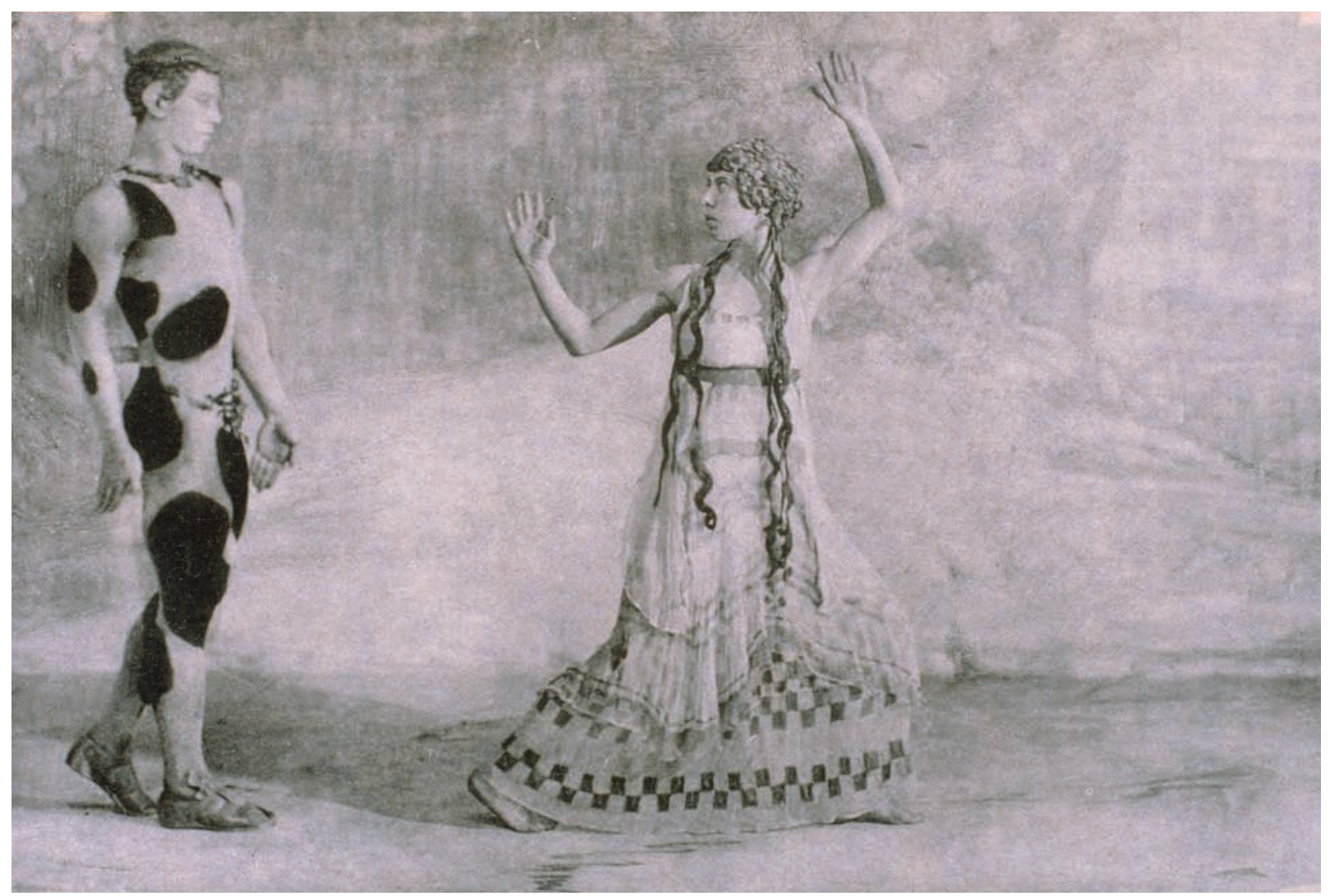

Fig. 4 : L'Après-midi d' un faune. Le faune (Nijinsky) et une nymphe (photographie Adolf de Meyer, 1912) (c) RMN

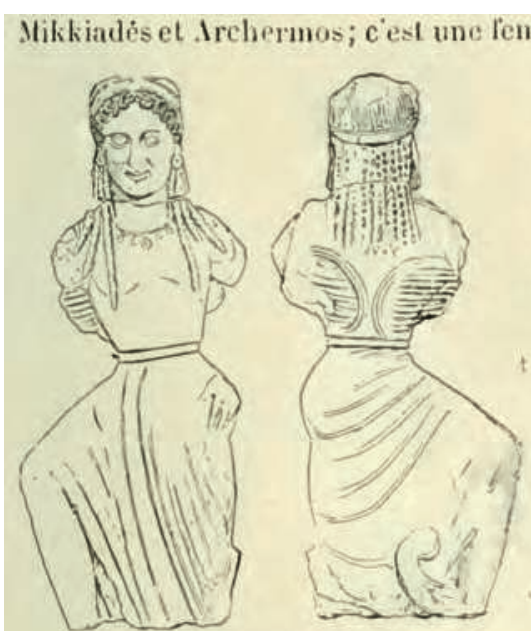

Fig. 235. - Irtemi* ailce. rétue trune longue tuniyue, roiffice d'un cliademe, ef qui porte des ailes aux éraules et aux talons; elle parait agenouillée, mai-plle euurt; le bras gauche tombant eot appuyé sur la hanche. le bras druit se portait en arant et se reptiait it partir dil coute, avec la main granle unverte (fis. 2334 ). il. Homolle y recomnait Irtémis ${ }^{61}$. C'est elle au-oi que dicril yl. Frohner.

Fig. 5 : Artémis ailée de Mikkiadès et Archermos (extrait du Dictionnaire des Antiquités grecques et romaines de Charles Daremberg et Edmond Saglio, Hachette, 1892, tome deuxième, première partie, fig. 2349, p. 133)

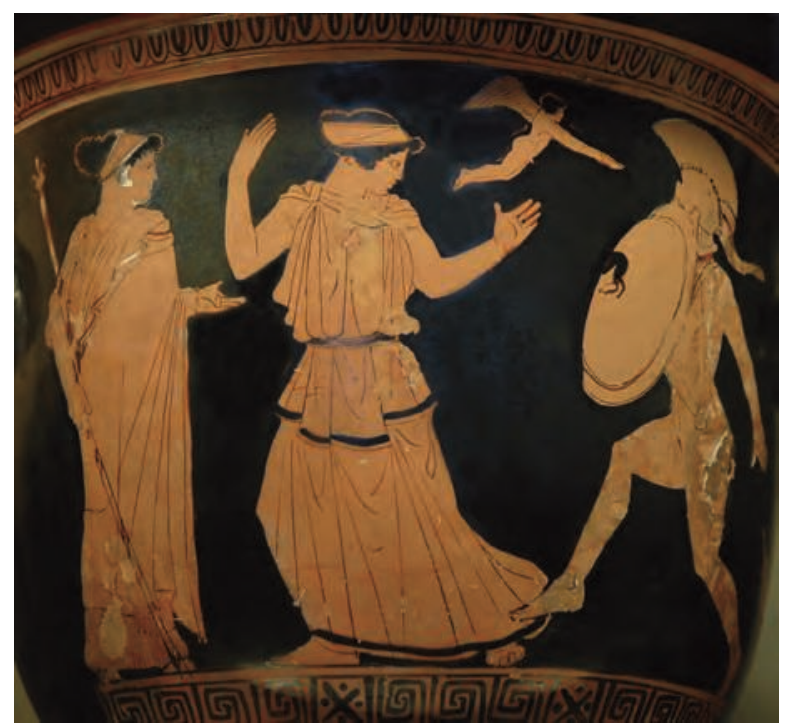

Fig. 5 bis : Ménélas poursuit Hélène. Détail d'un cratère attique à figures rouges, 450-440 av. J.-C.

(Louvre, département des Antiquités grecques, collection Campana) 
n'invitaient guère, en effet, au rappel, même allusif, du Faune mallarméen, debussyste et nijinskien, ce dernier fidèle au modèle antique jusqu'à embrasser avec passion une esthétique archaïsante et primitiviste. Le motif de la nymphe en fuite est profondément renouvelé et dramatisé par Proust, et sa source chorégraphique enfermée au fond d'une des nombreuses « cryptes » du roman.

Peut-être Nijinsky et Proust se rejoignent-ils pourtant ici par un même intérêt pour la survivance de certaines formes expressives - un intérêt qu'Aby Warburg problématise à la même époque à travers les Pathosformeln, où Ninfa, la Nymphe, joue d'ailleurs un rôle prépondérant 28 . « À son insu », autrement dit dans l'expression involontaire d'un fonds anthropologique dont les formes plastiques gardent la mémoire vive et souvent pathétique, M. de Charlus exprime, aussi bien qu'une jeune fille, la pantomime sans âge de la «terreur panique ».

\section{$*$}

Or la métabolisation, en 1915, de fragments de L'Après-midi d' un faune d'après le feuilleton de Bidou de 1912 n'était pas sans précédents génétiques. Nous avons ici pour guide notre mémoire de lecteur de la Recherche, frappé par un sentiment de familiarité à la lecture du texte de Bidou, comme si, par un étrange effet d'écho, nous y entendions celui de Proust. Voici un extrait de la première partie d'À l'ombre des jeunes filles en fleurs, au moment où, invité chez les Swann, le jeune héros rencontre pour la première fois Bergotte :

Ce premier jour où je le vis chez les parents de Gilberte, je racontai à Bergotte que j'avais entendu récemment la Berma dans Phèdre; il me dit que dans la scène où elle reste le bras levé à la hauteur de l'épaule - précisément une des scènes où on avait tant applaudi - elle avait su évoquer avec un art très noble des chefs-d'œuvre qu'elle n'avait peut-être d'ailleurs jamais vus, une Hespéride qui fait ce geste sur une métope d'Olympie, et aussi les belles vierges de l'ancien Érechthéion. «Ce peut être une divination, je me figure pourtant qu'elle va dans les musées. Ce serait intéressant à "repérer". [...] - Vous pensez aux Cariatides? demanda Swann (JF, I, p. 550).

Ces propos de Bergotte sur l'art de la Berma décalquent ceux d'Henry Bidou sur la chorégraphie de Nijinsky :
"Les deux nymphes qui entrent d'abord, la main levée à la hauteur de l'épaule, nous rappellent une Hespéride qui fait le même geste sur une métope d'Olympie. » L'introduction du motif, absent des brouillons (Cahier 24, fos 60-65 ros), s'est faite en deux étapes. Elle commence par un ajout sur la dactylographie du premier volume qui, rappelons-le, s'étendait alors jusqu'au premier séjour marin inclus, et dont la frappe était achevée à la fin du mois de juin 1912 :

[...] dans la scène où elle reste immobile un bras à demi levé $<$ revoit Thésée, la main levée à la hauteur de l'épaule - précisément la scène où on avait tant applaudi $\rightarrow$, elle avait eu le génie d'évoquer exactement et, avec un art très noble, et jusque dans la couleur générale eertaines statuettes < orantes> $<$ jeunes filles $>$ ioniennes que je connaissais par un des livres de lui que j'aimais le plus et où il leur adresse un de ses plus jolis eouplets <même les vierges de l'Érechthéion ancien Érechthéion qu'elle n'a peut-être d'ailleurs jamais vues. / Ce peut être une intuition divination. Je me figure pourtant qu'elle va dans les musées $>$ (NAF 16735, fo 139, p. « 549 bis »).

La «main levée à la hauteur de l'épaule » est un emprunt littéral à Bidou, tandis que les «vierges de l'ancien Érechthéion » reprennent en les précisant les «statues de l'Acropole »: si Proust évite de mentionner le nom de la colline d'Athènes c'est qu'il fait ici même (biffée, elle sera reprise quelques lignes plus bas) une allusion transparente à la « Prière sur l'Acropole » de Renan ${ }^{29}$, un des modèles affichés de Bergotte. On remarque aussi que la Berma n'est plus conçue sur le modèle d'une sorte de pythie tragique réincarnant, par « inttrition < divination>», l'art grec : instruit sur Nijinsky par Blanche et Bidou, Proust fait sienne - emprunte au danseur russe pour sa tragédienne fictive - l'idée du travail érudit de documentation préparatoire auquel se livre l'artiste (« Je me figure pourtant qu'elle va dans les musées »).

Au printemps de 1913, la scène fait partie du premier volume dont les quatre-vingt-quinze placards parviennent

28. Voir Georges Didi-Huberman, L'Image survivante. Histoire de l'art et temps des fantômes selon Aby Warburg, Paris, Minuit, 2002.

29. Voir $J F$, I, p. 550, n. 6. 
peu à peu à Proust. Celui où figure notre passage, le placard $\mathrm{n}^{\circ} 70$, lui arrive peu après le 3 juin ${ }^{30}$. On se trouve alors entre deux représentations du Faune, repris cette année-là au théâtre des Champs-Élysées les 17 et 23 mai, puis les 12,17 et 23 juin. Est-ce cette actualité qui rappelle à Proust l'article de Bidou qu'il a déjà utilisé l'année précédente pour la révision de ce passage sur la dactylographie ? Il en a certainement gardé un exemplaire :

Ce premier jour où je le vis chez les parents de Gilberte, je lui racontai que j'avais entendu récemment la Berma dans Phèdre; il me dit que dans la scène où elle revoit Thésée, la main levée <reste le bras levé> à la hauteur de l'épaule précisément la scène où on avait tant applaudi - elle avait ett te génie d'évoquer exactement, avee un art très noble, et jusque dans la couleur même, les vierges de l'aneien Érechthéion qu'elle n'a peut-être d'ailleurs jamais vues $<$ su évoquer avec un art très noble des chefs-d'œuvre qu'elle n'avait peut-être d'ailleurs jamais vus, une Hespéride qui fait ce geste sur une métope d'Olympie, et aussi les belles vierges de l'ancien Érecht[h]éion.> — Ce peut être une divination, je me figure pourtant qu'elle va dans les musées. - Ce sont $<$ Vous parlez $>$ des cariatides? demanda Swann (NAF 16753, plac. 70, col. 4) (voir fig. 6).

Proust fait réitérer l'image par le jeune héros quelques lignes plus loin : «Et je me disais : "Voilà <l'Hespéride d'Olympie ; voilà la sœur d'une > une de ces admirables orantes de l'Acropole ; voilà ce que c'est qu'un art noble"31 » (voir fig. 7). Ainsi, plutôt que la pose de la nymphe en fuite, l'écrivain aura d'abord retenu de L'Après-midi d'un faune l'attitude tranquille d'une autre nymphe, l'Hespéride, qui n'entretient aucun lien thématique direct avec le ballet (voir fig. 8). La scène est fixée sur le placard avant le 12 juillet 1913, date à laquelle Proust, d'après une lettre de Grasset, décide d'interrompre son premier volume au placard 59, le réduisant ainsi de plus du tiers ${ }^{32}$. Elle sera imprimée en 1914 sur les placards Grasset du Côté de Guermantes, qui serviront en partie à l'établissement par Gallimard des épreuves d'À l'ombre des jeunes filles en fleurs. Proust y apportera enfin quelques corrections de détail 33 .

L'inscription textuelle d'un minuscule fragment de l'article de Bidou et sa transposition contextuelle (de la chorégraphie à l'interprétation tragique) sont indiscernables pour le lecteur «moyen ». Faut-il alors parler de «plagiat»?
Non, puisque l'article est cité en mention, entre guillemets, comme propos rapporté. Il y a ainsi au moins un lecteur qui aurait pu en reconnaître l'origine, et s'y reconnaître, c'est évidemment Henry Bidou, qui aurait pu être amusé, flatté de voir sa prose et ses analyses mises dans la bouche du personnage du grand écrivain qu'admire le jeune héros, dans une transposition fidèle à l'esprit de sa chronique, celle d'un critique dramatique appréciant l'esthétique d'un danseur dans la mesure où elle « intéresse tous les interprètes tragiques ». Au moment où Proust corrige le placard, en juin 1913, la scène fait encore partie du livre qui doit sortir quelques mois plus tard, et on ne peut donc écarter l'intention, qui serait bien dans sa manière, d'une petite « carte de visite » adressée à Bidou, d'un hommage en forme de clin d'œil ${ }^{34}$. Dans cette hypothèse, ce n'est pas L'Après-midi d'un faune qui aurait intéressé Proust en 1912-1913 (et d'ailleurs rien ne certifie qu'il l'ait vu35), mais le style, le ton, la vision d'un journaliste.

Revenons pour finir à 1915 : pourquoi Proust introduisit-il finalement cette image frappante au moment de l'expulsion de Charlus ? Selon une addition apportée des années plus tard à la dactylographie de Sodome et

30. Ce placard ne porte pas de tampon, mais le précédent porte la date du 3 juin 1913, et le suivant, celle du 4.

31. NAF 16753, plac. 70, col. 5 .

32. Voir Corr., t. XIII, p. 396.

33. La planche en question du manuscrit morcelé des Jeunes filles, « No 15 », est conservée à la Beinecke Library (Yale University). Voir infra 1 'article de P. Wise.

34. Notons que Proust « tire » lui-même l'art chorégraphique du côté de l'art dramatique. Dans le Cahier 67, le narrateur enveloppe dans une même admiration deux exemples d' " artiste théâtral », l'actrice Sarah Bernhardt et le « danseur de génie » Nijinsky (fos 10-13 vos ; JF, I, « Esquisse III », p. 1001-1002) ; tous deux poursuivent le même but, produire « l'impression fugitive », donner à la fois « du ravissement et du regret ».

35. Le 13 mai 1912, Proust est «trop souffrant » pour voir au théâtre du Châtelet Le Dieu bleu, de Cocteau, F. de Madrazo et R. Hahn, qui ouvre avec d'autres pièces la saison « russe » (Corr., t. XI, p. 124), « si souffrant » qu'il manque sans doute le spectacle suivant, Thamar (ainsi que Carnaval, Le Prince Igor et Petrouchka) et le dîner offert chez Larue par Misia Edwards (ibid., p. 131, [le samedi 25 mai 1912]). Sur L'Après-midi d'un faune dont la répétition générale eut lieu le 28 mai et la première le 29, la correspondance est muette. Maurice Rostand affirme l'avoir vu en la compagnie de Proust (Confession d'un demi-siècle, Paris, La Jeune Parque, 1948, p. 175), Kolb et d'autres critiques plaçant l'événement plutôt en 1913, mais sans preuve (Corr., t. XII, p. 12 ; J.-M. Nectoux, « Proust et Nijinsky », art. cité, 2000, p. 79 et n. 5). 


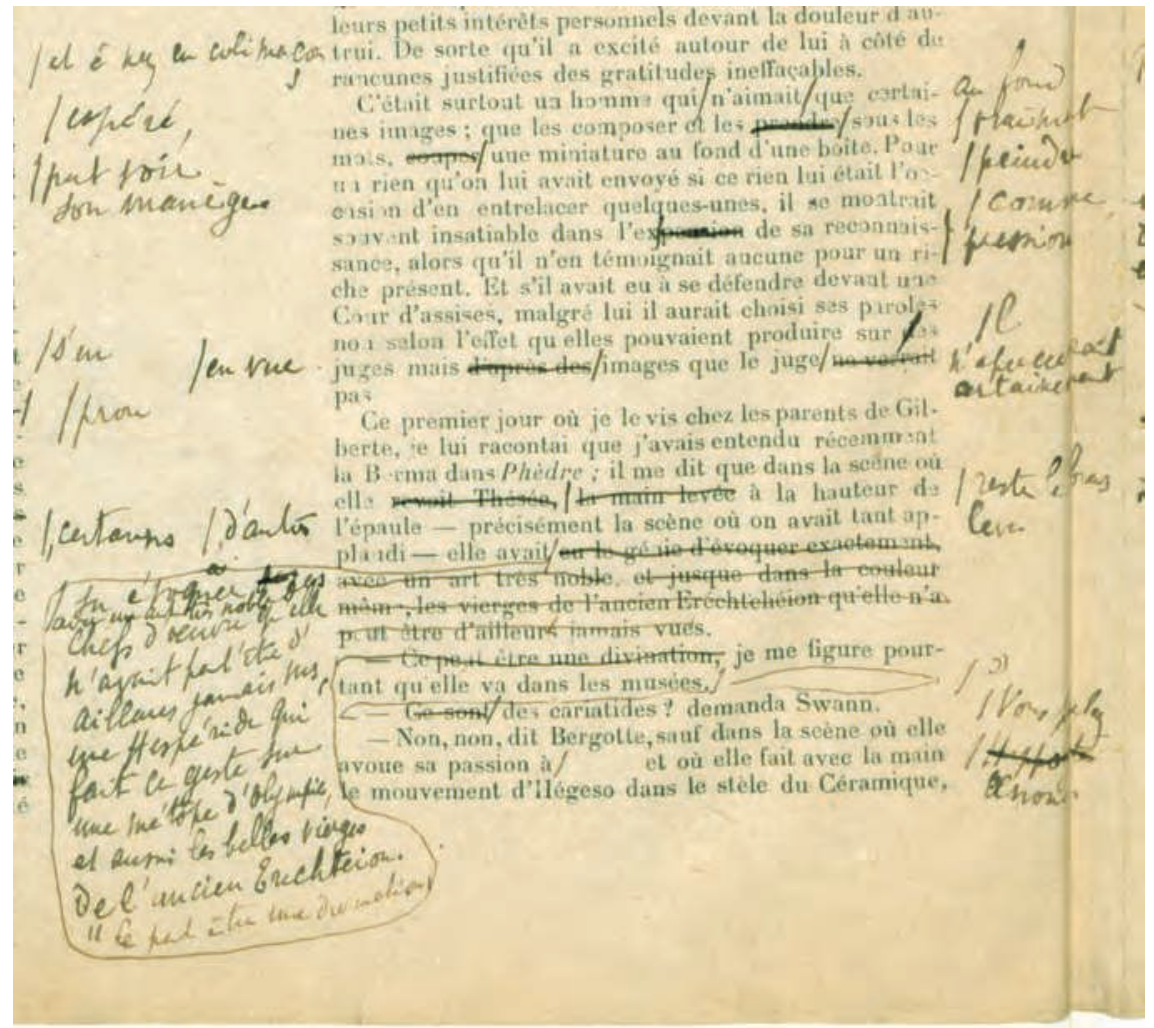

Fig. 6 : Placard Grasset du premier volume, n 70 , col. 4, détail (BnF, NAF 16753)

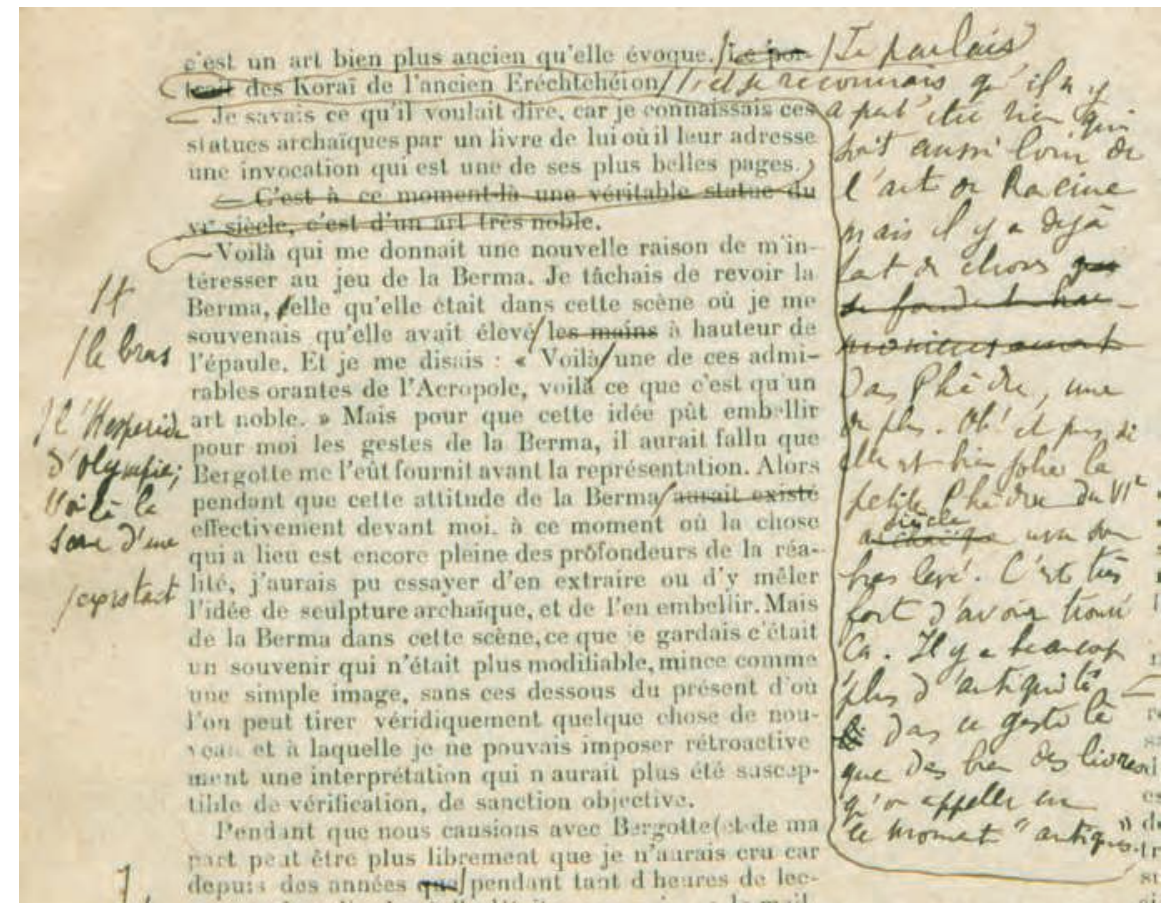

Fig. 7 : Placard Grasset du premier volume, $\mathrm{n}^{\circ}$ 70, col. 5, détail (BnF, NAF 16753)

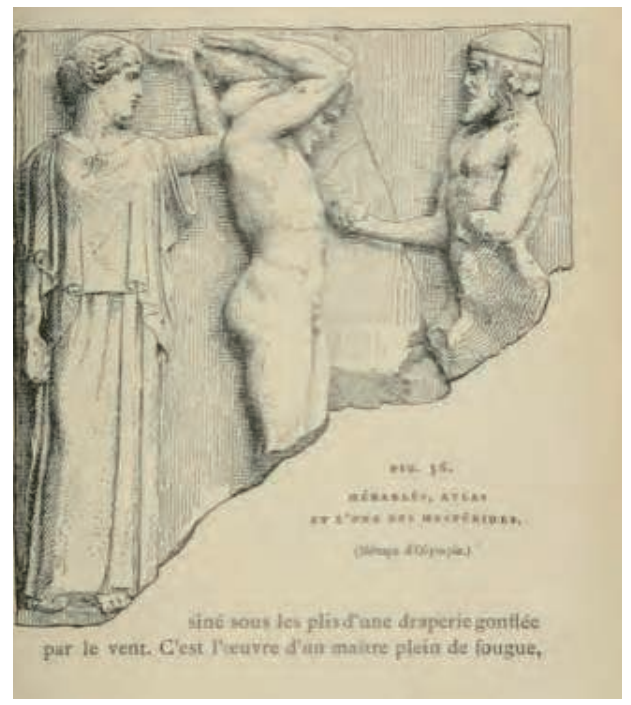

Fig. 8 : Hespéride sur une métope d’Olympie (d'après Maxime Collignon, Manuel d'archéologie grecque, Quantin, 1881, p. 179) 
Gomorrhe II (1922), M. de Charlus est le « manager » qui « sut mettre [la] virtuosité [de Morel] au service d'un sens artistique multiple et qui la décupla. Qu'on imagine quelque artiste purement adroit des Ballets russes, stylé, instruit, développé en tous sens par M. de Diaghilev36 » - qu'on imagine, autrement dit, le premier Nijinsky. Le parallèle entre le couple que forment Charlus et Morel et le couple Diaghilev-Nijinsky est quasi explicite ${ }^{37}$. S'agit-il alors d'une «pierre d'attente » qui aurait permis à certains lecteurs contemporains proches du milieu des Ballets russes d'entrevoir, en filigrane de l'exécution de Charlus, nymphe tragique et délaissée par son faune, la figure de Serge de Diaghilev, abandonné en 1914 par Nijinsky ? L'enquête génétique, loin de stabiliser l'interprétation, en déplie les horizons, et la rend toujours plus fuyante et feuilletée.

Retenons de cet exemple quelques traits du « style de genèse 38 » proustien : prélèvements ciblés dans un texte « source » d'éléments qui se trouvent reconfigurés par ellipse et condensation, ou qui sont cités en mention au sein de la fiction («Bergotte-Bidou» montrant en l'occurrence que l'exemple connu ${ }^{39}$ d' « Elstir-Mâle » n'est pas un cas isolé) ; absence de coïncidence entre la thématique du texte source et celle du ou des contextes d'inscription ; pluralité de ces inscriptions ; extension chronologique possible de l'usage d'un même texte source ; présence de visées allusives ; spécificité de ces visées. Enfin, l'extension au sein du roman de Proust de tels phénomènes complexes d'intertextualité est probablement sous-estimée, et constitue aujourd'hui un enjeu important de la recherche génétique. La numérisation extensive des sources (ouvrages, revues et presse) ne suffira pas à les repérer : les textes utilisés ne sont qu'exceptionnellement mentionnés dans les brouillons, et le travail d'écriture les déguise, les opacifie et les réinvente de façon imprévisible. Mais nous sommes, en puissance, les nouveaux destinataires de ces allusions perdues ${ }^{40}$ de la Recherche.

36. NAF 16740, fo 49 (SG II, III, p. 303).

37. Ce qu'avait déjà remarqué Jo Yoshida : voir « Proust et les Ballets russes : autour de Nijinsky », BIP, no 31, 2000, p. 60-61.

38. Sur cette notion problématisée par Anne Herschberg Pierrot, voir par exemple, de cet auteur, « Style de genèse et style d'auteur », Romantisme, $n^{\circ} 148$ (2010-2), p. 103-113.

39. Voir $J F$, II, p. $195-198$ et p. 198, n. 1.

40. J'emprunte l'expression à Antoine Compagnon (voir « L'allusion et le fait littéraire », dans L'Allusion dans la littérature, dir. Michel Murat, Paris, PUPS, 2000, p. 245). 
Nathalie Mauriac Dyer, directrice de recherche au CNRS (ITEM-ENS), s'est d'abord intéressée à la genèse et à l'histoire des tomes posthumes d'À la recherche du temps perdu (Proust inachevé. Le dossier «Albertine disparue », Champion, 2005). Actuellement responsable du Bulletin d'Informations proustiennes (Éditions Rue d'Ulm), elle pilote l'édition des Cahiers 1 à 75 de la Bibliothèque nationale de France (Brepols/BnF) où elle a notamment coédité les Cahier 54 (2008), 71 (2009) et 53 (2012). Derniers ouvrages codirigés : Proust aux brouillons (Brepols, 2011), Proust face à l' héritage du XIXe siècle. Tradition et métamorphose (PSN, 2012).

nathalie.mauriac@ens.fr

\section{Bidou, Bergotte, la Berma et les Ballets russes. Une enquête génétique}

La présence des Ballets russes (ou de toute autre manifestation artistique ou culturelle) dans le texte d'À la recherche du temps perdu ne se limite pas aux références explicites. L'écriture de Proust est constamment oblique et allusive. On suit ici, à partir d'un indice laissé par l'écrivain dans un cahier de brouillon, les usages, étalés entre 1912 et 1915, d'une chronique que le journaliste Henry Bidou avait consacrée à L'Après-midi d' un faune. Le caractère elliptique et condensé des réinscriptions de cet article, comme les contextes où elles interviennent, les rendent quasi indiscernables au lecteur contemporain de la Recherche : mais il est probable qu'elles constituent, en réalité, des allusions, dont nous pouvons chercher à identifier, parmi les contemporains de Proust, les destinataires possibles. En général, en l'absence de tout indice laissé par l'écrivain (ce qui est le cas le plus fréquent), ce sont des irrégularités locales (syntaxiques, lexicales, référentielles) qui peuvent nous alerter sur la présence d'un intertexte dans le texte publié et sur l'intérêt d'une enquête génétique.

The presence of the Ballets russes - or, for that matter, of any cultural or artistic event - in the text of $\grave{A}$ la recherche du temps perdu cannot be limited to its explicit mentions. Proust's style is constantly oblique and allusive. From a hint he left in a notebook, we explore how between 1912 and 1915 Proust used in his own text a newspaper chronicle by Henry Bidou on L'Après-midi d' un faune. Even though the condensed, elliptic character of its reinscriptions and the contexts in which they take place render them quite indiscernible to modern readers, it is likely that these were allusions destined to some of Proust's friends or contemporaries, whom we try to identify. When, as is usually the case, we have no such indication of a "source" from Proust's manuscripts, we can be alerted to the presence of some intertext, and the relevance of a genetic enquiry, by local disruptions in syntax, vocabulary or referential order.

Die Gegenwart des russischen Balletts (oder jeglicher anderer artistischer oder kultureller Ausdrucksformen) im Text Auf der Suche nach der verlorenen Zeit beschränkt sich nicht auf explizite Erwähnungen. Prousts Schreibstil ist stets indirekt und voller Anspielungen. Ausgehend von einem Hinweis des Autors in einem Notizbuch untersucht dieser Beitrag, wie sich Proust zwischen 1912 und 1915 einer dem L'Après-midi d' un faune gewidmeten Chronik des Journalisten Henry Bidou bedient. Die Vermerke auf diesen Artikel sind aufgrund ihres kondensierten, elliptischen Charakters und des Kontexts, in dem sie vorkommen, für den modernen Leser kaum zu erkennen. Wahrscheinlich handelt es sich um Anspielungen, die für einen von Prousts Freunden oder Zeitgenossenen bestimmt waren, dessen Identität wir hier nachgehen wollen. Wenn (wie meistens der Fall) keinerlei Hinweise des Autors vorhanden sind, sind es meist lokale Unregelmäßigkeiten (in der Syntax, in der Lexik, in der Referenz), die auf einen Intertext im veröffentlichten Text hinweisen und das Interesse an einer genetischen Untersuchung wecken können.
La presencia de los Ballets rusos (o de cualquier otra manifestación artística o cultural) en el texto de En busca del tiempo perdido, no se limita a las referencias explícitas. La escritura de Proust es permanentemente oblicua y alusiva. Se estudian aquí, a partir de un indicio dejado por el escritor en un cuaderno de borradores, la utilización, desplegada entre 1912 y 1915, de una crónica que el periodista Henry Bidou había dedicado a La siesta de un fauno. El carácter elíptico y condensado de las reinscripciones de este artículo, como los contextos en los que se producen, las vuelven casi indiscernibles para el lector contemporáneo de En busca..., pero es probable que, en realidad, constituyan alusiones y que sea posible identificar, entre los contemporáneos de Proust, a los destinatarios de las mismas. En general, y ante la ausencia de cualquier indicio dejado por el escritor (lo que ocurre con mayor frecuencia), son las irregularidades locales (sintácticas, léxicas, referenciales) las que pueden hacernos vislumbrar la presencia de un intertexto en el texto publicado y el interés de un análisis genético.

La presenza dei Balletti russi (o di qualsivoglia altro evento artistico o culturale) nel testo della Recherche du temps perdu non si limita ai riferimenti espliciti. La scrittura di Proust è, infatti, costantemente obliqua ed allusiva. A partire da un indizio affidato a un quaderno di abbozzi, il saggio esplora come Proust abbia reimpiegato a più riprese, fra il 1912 e il 1915, una cronaca che il giornalista Henry Bidou aveva dedicato a L'Après-midi d' un faune. Il carattere ellittico e condensato delle riscritture di tale articolo, come anche $\mathrm{i}$ contesti in cui esse si presentano, le rendono quasi irriconoscibili al lettore attuale della Recherche; ma è probabile che esse rispondessero tuttavia a una strategia allusiva, di cui giova identificare, fra i contemporanei di Proust, i possibili destinatari immediati. In generale, in assenza di indizi d'autore (ed è il caso più frequente), sono le irregolarità localizzate (di ordine sintattico, lessicale, referenziale) che possono segnalarci la presenza di un intertesto nel testo pubblicato e l'interesse di un'indagine genetica.

A presença dos Ballets Russos (ou de outra manifestação artística ou cultural) no texto de $\grave{A}$ la recherche du temps perdu não está limitada às referências explícitas. A escrita de Proust é sempre oblíqua e alusiva. Com pistas deixadas pelo escritor em um caderno de rascunhos, seguimos a sua utilização, entre 1912 e 1915, de uma crónica do jornalista Henry Bidou dedicada a L'Après-midi d'un faune. O carácter elíptico e condensado dos recursos a esse artigo, bem como o contexto em que ocorrem, tornam-nos quase indistinguíveis ao leitor contemporâneo da Recherche: mas é provável que eles sejam, na verdade, alusões de que os contemporâneos de Proust podiam identificar os possíveis destinatários. Em geral, na ausência de indícios deixados pelo escritor (caso mais comum), são irregularidades localizadas (sintácticas, lexicais, referenciais) que nos podem alertar para a presença de um intertexto no texto publicado e para o interesse de uma investigação genética. 